samples are drawn and centrifuged. RF and auto-antibodies to nuclear antigens anti-nuclear antibodies (ANA) and anti-double stranded DNA were determined in all the patients. All patients underwent X-rays of the hands and wrists.

Results: Anti-hnRNP A1 showed highly significant difference between study and control. Anti-hnRNP A2 showed significant difference between study and control. Conclusions: This study showed a high frequency of erosive arthropathy and autoantibody to both hnRNP antigens might become useful marker for joint involvement in SLE patients and identify SLE patients prone to develop joint damage

References:

[1] Pipili C, Sfritzeri A, Cholongitas E. Deforming arthropathy in systemic lupus erythematosus. Eur J Intern Med 2008;19(7):482-7.

[2] Pipili C, Sfritzeri A, Cholongitas E. Deforming arthropathy in SLE: review in the literature apropos of one case. Rheumatol Int 2009;29(10):1219-21.

[3] Hassfeld W, Steiner G, Graninger W, Witzmann G, Schweitzer H, Smolen JS. Autoantibody to the nuclear antigen RA33 - a marker for early rheumatoid arthritis.Br J Rheumatol 1993;86:19-203.

Disclosure of Interest: None declared

DOI: 10.1136/annrheumdis-2017-eular.4487

\section{FRI0666 INCREASED PREVALENCE OF SUBCLINICAL ATHEROSCLEROSIS IN MODERATE-SEVERE PLAQUE PSORIATIC PATIENTS}

N. Vegas-Revenga, J. Martín-Varillas, S. Armesto, M. Drake, V. Portilla, P. Fuentevilla, J. Rueda-Gotor, B. Atienza, L. Domínguez-Casas, C. FernándezDíaz, R. Blanco, M. González-Gay, A. Corrales. HUMV, Santander, Spain

Background: Recent studies suggest that plaque psoriasis may be a risk factor for major adverse cardiac events. This has important therapeutic implications for cardiovascular (CV) risk stratification and prevention in patients with severe psoriasis. For that reason surrogate markers of subclinical atherosclerosis and CV mortality such as carotid plaques (CP) have been studied by carotid US examination.

In most studies of psoriasis done before, the results have shown an increased prevalence of carotid plaques, but it is not always the rule.

Objectives: To compare the prevalence of CP between patients with moderatesevere psoriasis and the general population.

Methods: A cross-sectional study that included 40 patients with moderatesevere psoriasis (PASI $>10, \mathrm{BAS}>10 \%$ ), that fulfilled definitions for initiating treatment with a biological agent and 40 age-, sex- and traditional CV risk factorsmatched healthy control subjects. Patients with history of CV events, diabetes mellitus, and chronic kidney disease or body max index (BMI)>35 were excluded. Carotid ultrasonography was performed by a MyLab 70 scanner (Esaote; Genoa, Italy), then carotid plaque was defined according to the Manheim Consensus Conference criteria. Statistical analysis: Qualitative data were expressed as number and percentages and quantitative data as mean (SD). Student's t test or Mann-Whitney $U$ were used to compare continuous variables, as appropriate. Chi2 test or Fisher test were used for qualitative variables.

Results: The main data of the patients are summarized in the Table. It is important to highlight that it is based on a young population (mean age $<40$ years). The two groups did not present significant differences except for high sensitivity C-reactive protein (hsCRP)

As expected given the age of the group, CV risk measured by SCORE was low $(0 \%)$ with a mean of 0.2 us 0.15 . No patient had a high-very high CV risk as measured by SCORE ( $\geq 5 \%$ ).

Patients with psoriasis had a long-standing disease $(17.05 \pm 11.63$ years). The presence of carotid plaques was found in a total of 10 patients with plaque psoriasis (25\%), 5 of them had bilateral plaques) and one in the control group (2.5\%) without bilateral plaques), $p<0.003$.

\begin{tabular}{lccc}
\hline Variable & Plaque Psoriasis $(\mathrm{n}=40)$ & Controls $(\mathrm{n}=40)$ & $\mathrm{P}$ \\
\hline Age: Mean (SD) & $37,68(11.83)$ & $38.63(11.83)$ & 0.75 \\
Sex (Male): $\mathrm{n}(\%)$ & $18(45)$ & $18(45)$ & 1.0 \\
Psoriasis duration (years): Mean (SD) & $17.05(11.63)$ & $\mathrm{ND}$ & - \\
Psoriatic Arthritis: $\mathrm{n}(\%)$ & $11(27.5)$ & $\mathrm{ND}$ & - \\
HLA-B27 Positive: $\mathrm{n}(\%)$ & $5(13)$ & $\mathrm{ND}$ & - \\
BSA: Mean (SD) & $38.99(17.08)$ & $\mathrm{ND}$ & - \\
PASI: Mean (SD) & $19.33(8.89)$ & $\mathrm{ND}$ & - \\
hsCRP (mg/L): Mean (SD) & $3.26(3.31)$ & $1.69(2.62)$ & $\mathbf{0 . 0 0 1}$ \\
ESR (mm/h): Mean (SD) & $13.79(13.23)$ & $8.53(7.01)$ & 0.17 \\
Systolic hypertension (mmHg): Mean (SD) & $121.33(13.91)$ & $120.08(11.73)$ & 0.86 \\
Cholesterol (mg/dl): Mean (SD) & $196.08(34.31)$ & $193(36.42)$ & 0.69 \\
HDL-C (mg/dl): Mean (SD) & $55.58(17.05)$ & $63.88(20.7)$ & 0.051 \\
Smoking: $\mathrm{n}(\%)$ & $13(33)$ & $9(22.5)$ & 0.32 \\
Dyslipidemia: $\mathrm{n}(\%)$ & $21(53)$ & $17(42.5)$ & 0.37 \\
Arterial hypertension: $\mathrm{n}(\%)$ & $2(5)$ & $3(7.5)$ & 0.99 \\
Obesity (BMl>30) $\mathrm{n}(\%)$ & $7(18)$ & $3(7.5)$ & 0.18 \\
SCORE (\%): Mean (SD) & $0.2(0.46)$ & $0.15(0.43)$ & $\mathbf{0 . 5 5}$ \\
Carotid plaques & & & \\
Yes: $\mathrm{n}(\%)$ & $10(25)$ & $1(2.5)$ & $\mathbf{0 . 0 0 3}$ \\
Bilateral: $\mathrm{n}(\%)$ & $5(13)$ & $0(0)$ & $\mathbf{0 . 0 2}$
\end{tabular}

Conclusions: Moderate-severe psoriasis is associated with increased prevalence of carotid plaques.
Disclosure of Interest: None declared

DOI: 10.1136/annrheumdis-2017-eular.5320

\section{FRI0667 THE RECALL SURVEY: CAN ULTRASOUND AFFECTS CLINICIANS' DECISIONS ABOUT CHANGING TREATMENT IN RA?}

O. Epis ${ }^{1}$, P. Macchioni ${ }^{2}$, A. Delle Sedie ${ }^{3}$, M. Canzoni ${ }^{4}$, E. Filippucci ${ }^{5}$ A. lagnocco ${ }^{6}$, S. Tropea ${ }^{7}$, C.F. Bonali ${ }^{8} .{ }^{1}$ Rheumatology Unit, Ospedale Niguarda Milano, Milano; ${ }^{2}$ Rheumatology Unit, Reggio Emilia Hospital, Reggio Emilia; ${ }^{3}$ Rheumatology Unit, Università di Pisa, Pisa; ${ }^{4}$ Local Health Unit (ASL) Rome 1, Rome; ${ }^{5}$ Rheumatology Department, Università Politecnica delle Marche, Jesi (AN); ${ }^{6}$ Academic Rheumatology Unit, Università degli Studi di Torino, Torino; ${ }^{7}$ ASP7, Busacca Hospital, Ragusa; ${ }^{8}$ Rheumatology Unit, Ospedale San Paolo, Bari, Italy

Background: In Rheumatoid Arthritis (RA) treatment response is assessed using standard clinical disease activity measure. However ultrasound (US) is able to show subclinical synovitis in patients (pts) with RA who are in clinical remission (CR); further studies are still required to delineate the impact of US findings in the management of RA pts in daily clinical practice.

Objectives: to investigate the influence of US on the clinicians' treatment choices in pts with RA.

Methods: in 2015 an educational event (RECALL Survey) focused on the added value of US in RA pts was held in 22 rheumatology centers in Italy.In every center, the local rheumatologists provided RA pts to be examined by US. Pts signed an informed consent and a brief history of them was collected by the local rheumatologists (previous and current therapy, DAS28, HAQ score). Bilateral US examinations of wrists, metacarpophalangeal (MCP) and metatarsophalangeal (MTP) joints were performed by rheumatologists expert in US, to assess synovitis (joint effusion, synovial proliferation, and power Doppler (PD) signal), and bone erosions, using a Logiq E R7, General Electrics, with a 4.2-13 MHz linear probe. All US findings were scored using a 4 degree semiquantitative scoring system. Results: 465 pts were evaluated. Clinicians, after US evaluations, changed therapy in $23.7 \%$ of pts, did not change therapy in $35.5 \%$ of pts.In general changes of therapy tended to be made by clinicians when joint effusion or power Doppler signal were present (table 1-2). The presence of erosion did not influence the clinicians' decisions.

Table 1. Changes of therapy and joint effusion

\begin{tabular}{lcccc}
\hline & & \multicolumn{3}{c}{ Joint Effusion } \\
\cline { 3 - 5 } & & Score $\leq 0$ & Score $>0$ & Total \\
\hline Change in therapy & No & 86 & 79 & 165 \\
& Yes & 11 & 99 & 110 \\
& Total & 97 & 178 & 275 \\
\hline
\end{tabular}

Table 2. changes of therapy and Power Doppler

\begin{tabular}{lcccc}
\hline & & \multicolumn{3}{c}{ Power Doppler Signal } \\
\cline { 3 - 5 } & & Score $\leq 0$ & Score $>0$ & Total \\
\hline Change in therapy & No & 129 & 36 & 165 \\
& Yes & 24 & 86 & 110 \\
& Total & 153 & 122 & 275 \\
\hline
\end{tabular}

Conclusions: Ultrasound may be an useful tool in daily rheumatologic practice to help clinicians make decisions about how to treat patients with RA. US results, especially joint effusion and Power Doppler signal, may influence the choice of clinicians to modify a patient's treatment regime.

Disclosure of Interest: None declared

DOI: 10.1136/annrheumdis-2017-eular.4090

\section{FRI0668 SONOGRAPHIC AND ANATOMICAL DESCRIPTION OF THE SUBTALAR JOINT}

P. Mandl ${ }^{1}$, D. Bong ${ }^{2}$, P.V. Balint ${ }^{3}$, H.B. Hammer ${ }^{4}$, M. Miguel ${ }^{5}$, E. Naredo ${ }^{6}$, I. Möller ${ }^{2}$ on behalf of Anatomy for the Image. ${ }^{1}$ Rheumatology, Medical University of Vienna, Vienna, Austria; ${ }^{2}$ Rheumatology, Universitat de Barcelona, Instituto Poal de Reumatologia, Barcelona, Spain; ${ }^{3}$ Rheumatology, National Institute of Rheumatology and Physiotherapy, Budapest, Hungary;

${ }^{4}$ Rheumatology, Diakonhjemmet Hospital, Oslo, Norway; ${ }^{5}$ Pathology and Experimental Therapeutics, Universitat de Barcelona, Barcelona;

${ }^{6}$ Rheumatology, Joint and Bone Research Unit. Hospital Universitario Fundación Jiménez Díaz and Autónoma University, Madrid, Spain

Background: The subtalar joint is commonly affected in many rheumatic and musculoskeletal diseases; however, subtalar joint involvement is often neglected or missed during clinical examination due to the fact that the joint is difficult to examine and most clinicians have a limited understanding of its anatomy.

Objectives: To provide a detailed anatomical and US description of the subtalar joint, a single joint that, anatomically, is divided into two separate compartments: the anterior subtalar joint (ASTJ) and the posterior subtalar joint (PSTJ).

Methods: Cadaver specimens of the ankle and foot were examined in detail by ultrasound (US) by rheumatologist experts in musculoskeletal US. The ASTJ of all 
the specimens were injected with colored latex while the PSTJs were respectively injected with $1 \mathrm{ml}$ of latex of a contrasting color under US guidance. Following the injections, the joints were frozen and cut into 2 centimeter sections from medial to lateral in the sagittal plane. Sections were examined independently by three authors for the presence of latex within the ASTJ, PSTJ and, also, to look for the presence of extra-articular latex extravasation or the spread of latex to adjacent articulations by means of a communication with the ankle joint or the adjacent portion of the subtalar joint.

Results: Six cryopreserved intact ankle-foot specimens from three male and three female cadavers (two right and four left), with a mean age of 74 years (range, 66-80 years) were studied. A recommended list of standardized scanning technique which allows sonographers to evaluate both compartments of the subtalar joint (ASTJ and PSTJ) from the medial, lateral and posterior aspect were developed. All of the specimens $(6 / 6,100 \%)$ contained the appropriate colored latex in the appropriate subtalar joint compartment with minimal leakage into the surrounding soft tissues (Figure 1$)$. Of note, five of the six $(5 / 6,83 \%)$ specimens revealed communication between the PSTJ and the posterior tibiotalar joint. There was no communication between the ASTJ and the tibiotalar joint, nor was there evidence of spread of the latex, i.e. communication, from one subtalar joint compartment to the other.

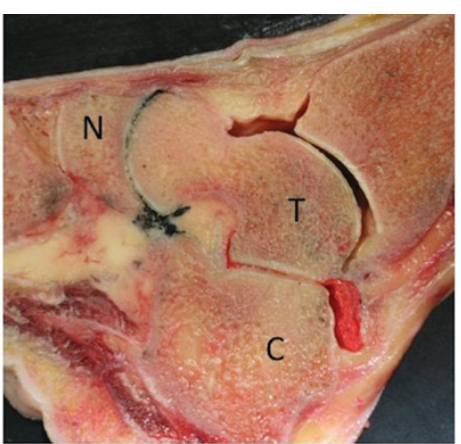

Figure 1. Anatomical specimen showing the anterior subtalar joint (filled with green latex) anc the posterior subtalar joint (filled with red latex);

$\mathrm{C}$ : calcaneus, $\mathrm{N}$ : navicular, $\mathrm{T}$ : talus

Conclusions: Lack of communication between the ASTJ and the PSTJ and the communication between the PSTJ and the posterior recess of the tibiotalar joint are compatible with other studies. Our study highlights the importance of employing cadaver specimens in musculoskeletal ultrasound, and presents a number of options for US imaging of both the ASTJ and PSTJ that also provide access to these distinct compartments for the purpose of aspiration and injection.

Disclosure of Interest: None declared

DOI: 10.1136/annrheumdis-2017-eular.2675

\section{FRI0669 VALIDITY OF SEVEN-JOINT VERSUS SIMPLIFIED TWELVE-JOINT ULTRASONOGRAPHY SCORING SYSTEMS IN ASSESSMENT OF RHEUMATOID ARTHRITIS ACTIVITY}

R. El-Gohary ${ }^{1}$, A. Ahmed ${ }^{1}$, A. Khalil ${ }^{2}$, H. El-Gendy ${ }^{1}$, K. Gado ${ }^{1}$. ${ }^{1}$ Internal Medicine, Rheumatology \& Clinical Immunology Subspeciality, Kasr Alainy; ${ }^{2} \mathrm{New}$ Kasr El-Aini Teaching Hospital, Cairo, Egypt

Background: Musculoskeletal ultrasonography imposes itself as reliable tool for the disease activity assessment of rheumatoid arthritis (RA) being more sensitive than clinical in detection of synovitis. There is no consensus on the exact joint number should be examined. Naredo et al. developed reduced 12-joint count ${ }^{1}$ while Backhaus et al. scored only seven small joints ${ }^{2}$. Although both were found to reflect disease activity it is suitable in a busy clinic to assess the lowest joint count.

Objectives: To investigate the validity of 7-joint ultrasonography (US7) scores in assessment of disease activity in Egyptian RA patients compared with simplified 12-joint ultrasonography (US12) scores and correlate both to composite disease activity indices.

Methods: Fifty Egyptian RA adult patients diagnosed according to the ACR1987 criteria were subjected to detailed history, 28 tender \& swollen joint counts (TJC, SJC). The disease activity was assessed by calculating DAS28-CRP, SDAI and CDAI. The Ultrasonographic assessment was performed using a LOGIQP6 with 10-13 MHz broadband linear array transducer by one well-trained blinded rheumatologist. The synovial hypertrophy on GS \& PD images were graded using a semi-quantitative $0-3$ scale. The simplified US12 was performed as originally described ${ }^{1}$. However the GS \& PD synovitis were computed in two separate scores instead of one. The US7 was performed at the clinically dominant side as described by Backhaus et $\mathrm{al}^{2}$. Two Sum-US7 scores were added; Sum (GS)-US7 after grading the GS-tenosynovitis and Sum (PD)-US7. Ultrasonography inter/intraobserver reliability was evaluated on 40 stored images using randomization and blinded techniques. The study was approved by the local ethics committee.

Results: The studied population had mean disease duration of $89 \pm 60$ months.
$62 \%$ were using more than one DMARD. They showed moderate disease activity as defined by DAS28, SDAI and CDAI (mean 3.7 $\pm 1.4,19.9 \pm 14.9$ \& $17.9 \pm 13.3$ respectively). The GS-scores showed no correlation with disease activity parameters. PD-US12, PD (synovitis)-US7 and Sum (PD)-US7 significantly correlated with the SJC, CRP levels (P values $0.03,0.04$ and 0.01$)$ and DAS28 (P values $0.02,0.009$ and 0.001 ), furthermore Sum (PD)-US7 correlated with SDAI and CDAI ( $P$ values $0.013 \& 0.042$ respectively). Correlation coefficient between the different ultrasonographic scores showed no differance. The ultrasonography reliability showed significant agreement $(P<0.001)$. Kappa values were 0.80 \& 0.96 for GS \& PD interobserver reliability and 0.96 \& 1.0 for GS \& PD intraobserver reliability.

Conclusions: Our results confirm the validity of PD-scores in assessment of disease activity. However they make the significance of GS questionable. The Sum-(PD) US7 seems to perform better than the original one, it was strongly correlated with CRP and with the three disease composite indices. The studied scores correlated with each other favoring the use of less time consuming US7score.

[1] Naredo E, et al. Validity, reproducibility, and responsiveness of a twelve-joint simplified power Doppler ultrasonographic assessment of joint inflammation in rheumatoid arthritis. Arthritis Care Res. 2008;59(4):515-22.

[2] Backhaus $M$, et al. Evaluation of a novel 7-joint ultrasound score in daily rheumatologic practice: A pilot project. Arthritis Care Res. 2009;61(9):1194201.

Disclosure of Interest: None declared

DOI: 10.1136/annrheumdis-2017-eular.3256

\section{FRI0670 THE COURSE OF SUBCLINICAL JOINT INFLAMMATION DURING PROGRESSION FROM ARTHRALGIA TO CLINICALLY DETECTABLE ARTHRITIS, A LONGITUDINAL MRI STUDY}

R.M. Ten Brinck, H.W. van Steenbergen, L. Mangnus, A.H. van der Helm-van Mil. Rheumatology, Leiden University Medical Centre, Leiden, Netherlands

Background: Subclinical joint inflammation in the phase of arthralgia preceding RA can comprise synovitis, bone marrow edema (BME) and/or tenosynovitis. It is unknown in what time order the different tissues in a joint become inflamed or progress with inflammation. It has been postulated that synovitis is an initial process that is succeeded by bone involvement ("outside-in hypothesis" presuming that synovitis precedes BME and erosions). Alternatively, it is suggested that inflammatory cells in the bone marrow migrate via bone pores to the synovium, promoting synovitis ("inside-out", then BME precedes synovitis). Thirdly, these processes can occur or progress simultaneously. Serial MRI studies can reveal time-relationships. This longitudinal MRI study on patient-level determined the course of joint inflammation during progression from arthralgia to clinical arthritis. Objectives: To increase the comprehension on the course of MRI-detected subclinical joint inflammation during progression from arthralgia to clinical arthritis, both in ACPA-positive and ACPA-negative arthritis patients.

Methods: We longitudinally followed 29 patients that all progressed from Clinically Suspect Arthralgia to clinical arthritis. 1.5T MRI on hand and foot joints was performed at presentation with arthralgia and subsequently at the development of clinical arthritis. MRIs were evaluated for BME, synovitis and tenosynovitis (summed in the total inflammation score) and erosions by three readers (ICCs $0.98,0.96$ and 0.97 ) that were blind to clinical data and the order in time. Analyses were repeated in arthritis patients that fulfilled the 2010 criteria for rheumatoid arthritis, and in ACPA-positive and ACPA-negative patients separately.

Results: At presentation with arthralgia the mean age was 43 years, $72 \%$ was female, and $28 \%$ was ACPA-positive. Median duration to clinical arthritis was 17 weeks. At the time of arthritis development $65 \%$ fulfilled the 2010 -criteria. During progression from arthralgia to clinical arthritis the median total inflammation score increased from 4.5 (IQR 2.8-7.8) to 6.0 (IQR 2.0-13.5), $\mathrm{p}=0.01$. The BME score increased over time $(p=0.04)$, as did the synovitis score $(p=0.002)$. The tenosynovitis and erosion scores increased as well, though not statistically significant $(p=0.10$ and $p=0.07$ respectively). Analyses within the patients that developed 2010-RA revealed that BME $(p=0.047)$, synovitis $(p=0.005)$ and tenosynovitis $(p=0.004)$ all increased significantly, in contrast to the erosion score. At presentation with arthralgia, BME and synovitis scores were higher in ACPA-positive than in ACPA-negative patients; during progression to clinical arthritis the different types of inflammation increased similarly in ACPA-positive and ACPA-negative patients.

Conclusions: During progression from arthralgia to clinically evident rheumatoid arthritis, BME, synovitis as well as tenosynovitis progress simultaneously.

Disclosure of Interest: None declared

DOI: 10.1136/annrheumdis-2017-eular.2908 